Journal of Computer Science 8 (3): 342-347, 2012

ISSN 1549-3636

(C) 2012 Science Publications

\title{
Wetland Ontology Modeling to Apply the Context-Aware Technology for Application Service Environment
}

\author{
Gwi-Hwan, J.I. and O.H. Ryum-Duck \\ Department of Computer Science and Information Engineering, \\ Chung-Ju National University, Chungju-Si, South Korea
}

\begin{abstract}
Problem statement: Due to recent advances in the IT industry, the importance of ontology has increased as a tool of knowledge representation at home or abroad for implementation of intelligent and personalized service system such as next generation semantic web environment and ubiquitous computing environment. But, although the importance of wetlands are being recognized, systematic management is still not enough due to insufficiency of financial or professional management personnel. Conclusion/Recommendations: Therefore, this study aims to analyze the information on the wetlands environment which provides a variety of human comfort, designs wetland environment context awareness data model by detecting lexical meaning and relationship between words using ontology techniques for the application of wetland environment and presents methods for systematic management of the wetland environment.
\end{abstract}

Key words: Context-aware, ontology model, wetland environment, semantic web, water-quality automatic measurement monitoring location, Ubiquitous Sensor Network (USN), natural environment, systematic management

\section{INTRODUCTION}

Wetlands is a wet ground surrounded by rivers, ponds, swamps and water remains all the time due to natural environment around and rich in natural resources and it has a variety of benefits such as flood control, slope erosion control, animal habitat and ground water supplies, water quality improvement, prevention of global warming. As a target of management at a national level, it will require active conservation activities Kwater Environmental Kit.

Wetlands are treated as a national problem around the world but, the management does not have a special system compared to interest and importance due to lack of finances and professional managers. Therefore, effective wetland management system even with minimum finances is urgently needed. In addition, systematic method of water quality such as each Ubiquitous Sensor Network (USN) service domain, ontology-based context awareness, production, management technology development appropriate for the type of service is required for the development of USN middleware-based services with recent changes in the trends of next-generation semantic web environment.

A variety of environmental conditions such as eutrophication, algal blooms in the wetland environment can make a difference in the probability of change and occurrence in the evaluation of plural items that exist in each circumstance. For such reasons, terms are defined in part, an awareness of plural situations that each item has is, in reality, evaluated only by a few experts, an the baseline of data is defined differently by national unit or by researcher Natlonal Institute of Environmental Research Environmental Kit. Therefore, context awareness reasoning, where similar service search is available, is needed in terms of application of service by use of systematically-defined knowledge, even though perfectly matched service to situation that one wants to find is not defined (Choi and Park, 2009). To this end, this study defines underlying ontology as various water elements across the wetlands. This also designs wetlands environment situation awareness ontology models and presents its application methods for efficient management of water environment to make it possible to be convenient and quick in analysis of the wetland environment and be precise in the detection of abnormal data and be immediate in the context awareness even without specialized knowledge.

As for configuration explains related researches; 3 designs situation awareness model by the definition and data analysis on a variety of sensors and items for the recognition of wetland environment; and then presents forthcoming research directions and comes into a conclusion.

Corresponding Author: Gwi-Hwan, J.I., Department of Computer Science and Information Engineering,

Chung-Ju National University, Chungju-Si, South Korea 


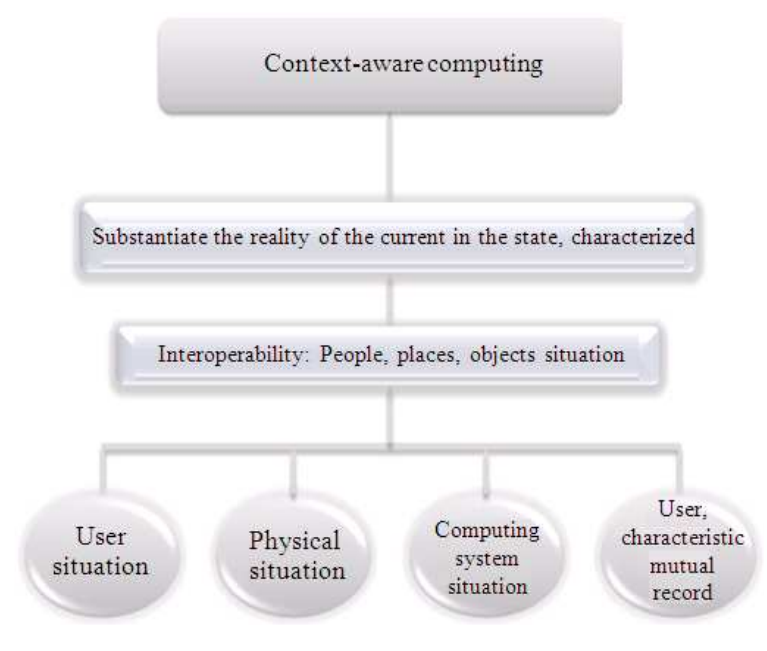

Fig. 1: Context-aware structure

Related research: Ontology consists of words and relationships as formal and explicit specification to conceptualize some areas of interest. And words related to a specific domain are represented hierarchically and additionally includes inference rules to extend this. Thereby web-based knowledge processing, knowledge sharing between applications and the reuse is possible (Mahesh, 1996) and it gives us the infrastructure of information sharing to allow us systematic sharing of information (Park and Rarn, 2004).

Ontology-based search system has advantages: it can alleviate user's repeated searches as a hierarchical search; it is easy to retrieve various information on the status of wetlands which is the top-level domain in this study through definition of relationship between terms and associated search. An ontology that makes a hierarchical classification of words on a specific subject can be utilized in various fields and can be provided as a basis for ontology modeling in varied environmental field in the upcoming years. Ontology is currently under development in many areas and automated search support for user needs has been studied by using relationship between upper classes (Srinivasan et al., 2006; Ranganathan and Campbell, 2003). Such ontology establishment methods are: (a) use of existing thesaurus or dictionaries (Lim et al., 2003) (b) extension of base ontology by using distribution of words obtained as a result of text analysis (Lim et al., 2004). In context awareness computing, model is required to access, mange and express situational information. In such context awareness model, the former method, ontology-based approach can build the knowledge base which can be immediately available without any extra work as public data such as legal standards and already publicized items are easily obtained from official agencies in the nature of wetland environment. This study uses the former method which is possibly available to develop ontology with limited time and personnel, to build ontology model.

\section{Wetland environment analysis-based ontology model design: \\ Configuration of ontology context awareness} system: Ontology-based context awareness technique requires clear definition of what the first thing is first to provide information with use of terms that computer understands. Technical measure which can express every situation in the real world is required to make real situation in the virtual space into information by linking the real space and virtual space and, by using this to provide intelligent user-centric services. Based on this, general ontology context awareness system configuration is shown in (Fig. 1).

Figure 1 is a structure map which is composed of four kinds of general context information and its subelements are user's physical conditions, computer system, user characteristics interaction history. This indicates that as for ontology design, situation model should be established based on interoperability of subelements such as users' status and physical situation, i.e., sensor, overall computer, user, specific interaction history.

Wetlands environmental data analysis: What should be preceded prior to the ontology modeling is to analyze various data of wetland environment and classify the relationship between terms. Thereafter, ontology model is to be designed as to more than 60 items of investigation items related to the current water quality and a variety of situations of wetlands.

This study presents context awareness ontology model for eutrophication and algae which heavily influences the wetland water quality with more than 60 water quality standards and collectible data from automated water quality monitoring, that is, automatic continuous measurement of water pollution monitoring in the river or lakes. Each test item mentioned has shown a correlation between items and the relationship between the terms has a direct impact on the upper domain of this study: eutrophication, prediction of tidal currents, algal blooms, sewage flows, which may act as a combination. First, prediction of tidal currents has four steps separately: watch, alarm, hypertrephied, lifting based on Chlorophyll-a concentration and cyanobacterial cells. The criteria of eutrophication includes T-P, T-N, chlorophyll a and transparency. The criteria of eutrophication depends on nation, lake and researcher, ontologization of the criteria of eutrophication to uniform numerical value is an 
essential part for situation awareness service. Among various criteria of wetland environment, this study refers to credible and authorized reference materials for situational information such as algae alert and eutrophication and focuses on the situation of eutrophication since the effects of eutrophication are general and widespread.

Figure 2 shows positions of water quality monitoring network and current status of sensor reserve at the Water Purity Control Office.

Each sensor of Water Quality Monitoring Network has different type of sensor at each location, Each item is modeled into instances under position class. If any properties that belongs to the criteria between algae phenomenon and eutrophication are compared to sensors that automatic monitoring device has, the status is not constant.

For example, as shown in (Fig. 2), Noryangjin must determine eutrophication through multiple situations by two sensors: T-P and T-N. As there exist two evaluation criteria like single evaluation and multiple evaluation, Noryangjin is not an impossible area to assess eutrophication unlikely as in Jamsil and Gumgang although accuracy may be problematic. If properties that belong to each situation and definition of the relationship and its association are designed through modeling, it is possible to determine whether situations are recognizable and judge depending on conditions.

\section{Wetlands environmental contest awareness ontology model: \\ Basic ontology: This defines a direct relationship with} data by building key map for the connection and extension of relationship between information and conceptual framework based on investigated data. First, the most upper domain is defined as wetland environment and dictionary definition of terminologies which has a lack of public awareness should be given. In addition, properties that have a big impact on seasonal or geographical location and the overall situation of the wetland environment together with the data collection of each and every situation via sensors are put into sub-category and instances suitable for the characteristics of each class are set and grouped.

When building ontology in situation awareness computing as a model for accessing, managing and expressing situational information, a designer determines a small number of nodes in the high level and based on this, builds and extends ontology, which is called basic ontology as shown in (Fig. 3).

In (Fig. 3), each instance of sensor class is multiply included in each property of location class separately, sensor's instance in modeling explicit domain ontology of "include" which forms inclusion relation for each property of location.

Figure 3 is a simplified event situation of wetland environment and has higher class such as sensor, status, season and region. Each higher class should be able to consider expansion and express complex and fragmented key map on schema implementation for an optimized management of wetland environment through reference or inheritance for ontology characteristics. As for such complex ontology, the old diagram edit methods have constraints, Class tree should be designed to regulate the level of information.

Wetlands environmental ontology model design: Comprehensively, information on the properties and conditions mentioned in this study should be stratified and, as shown in (Fig. 3) the type of sensors between classes, that is, measurement data for each item, measurement location and the situations that can occur should be represented on the ontology model structure and models shown in (Fig. 4) are designed.

The arrows represent the relationship between classes and the relevance of each plural item are grouped as a large circle.

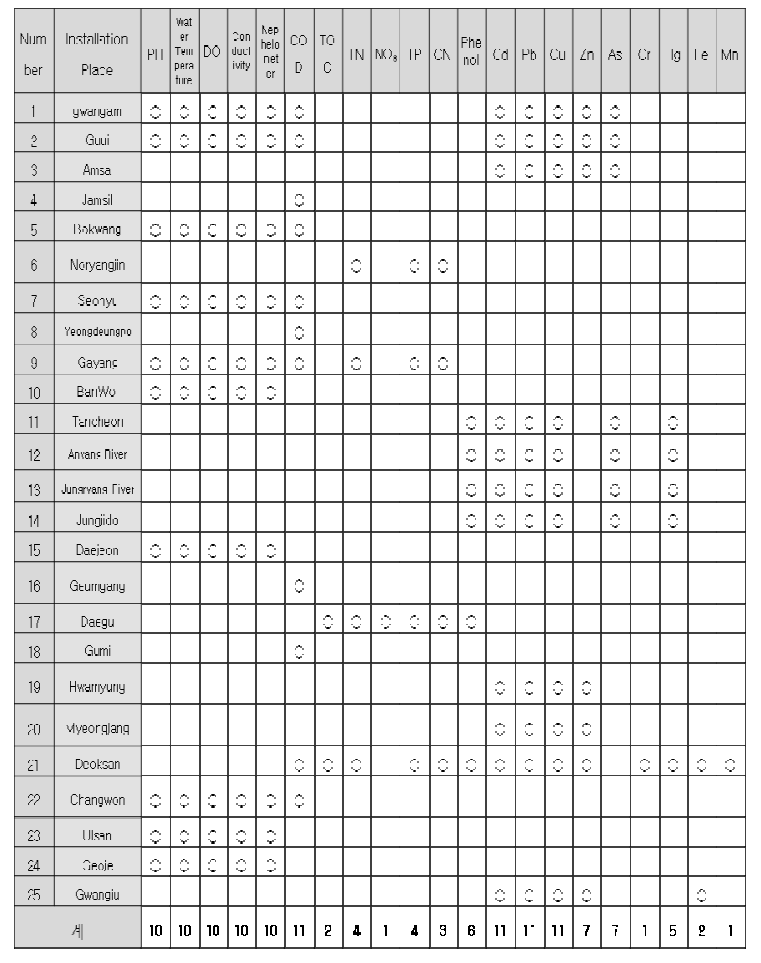

Fig. 2: Water-quality automatic measurement monitoring location and Measurement sensors in Korea 


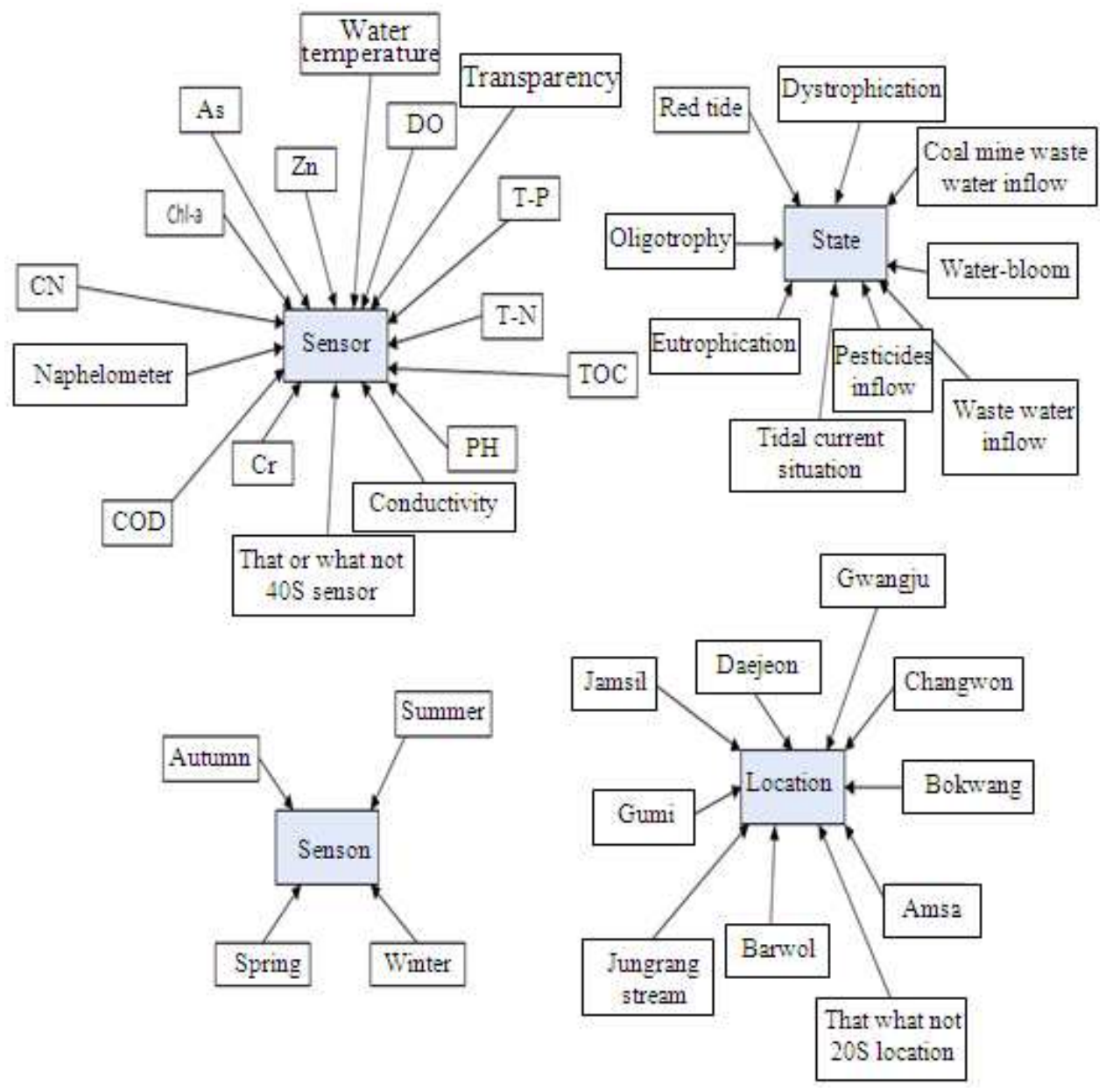

Fig. 3: Wetland environment base ontology

Table 1: Verb ontology signification relationships

\begin{tabular}{ll}
\hline Classification & Signification relationships \\
\hline Verb ontology & $\begin{array}{l}\text { Compare, search, warn, measurement, } \\
\text { detection, occurrence, increase, decrease }\end{array}$ \\
\hline
\end{tabular}

The best upper class and sub classes like sensor, location, status, season were set and each of such four classes include subclasses as shown in (Fig. 4).

Each property in the sub-class modeling uses verbal ontology key words such as "include", "compare", "measure", "exceed" to describe the relationship between property, deduce a situation when a certain situation occurs and provides already formulated engineering measures suitable for step-by-step situational alarm of eutrophication or situation step.

Context inference through verbal ontology: As a representation of domain ontology, verbal ontology makes a modeling of verbal words into a stratified structure and is able to deduce an appropriate service by

situational reasoning in the event of certain circumstances. The key words that configure wetland environment situation awareness ontology model are set like (Table 1) in the below based on the criteria of eutrophication.

As the sensors of Water Quality Automatic Monitoring Network have different reserve items on each location, "include" is set in the location class.

Figure 4 separates two layers(upper and lower) as a conceptualized ontology and context-awareness service can be provided by making a modeling of low-level sensor information into ontology-based information and converting it into upper-level information of the situation. As mentioned the sensor locations shown in (Fig. 2) are put on location class of (Fig. 4) and has been modeled to determine abnormalities of the data and locations based on legal standards in the event of situation search compared to the data each sensor receives shown in (Fig. 4). 


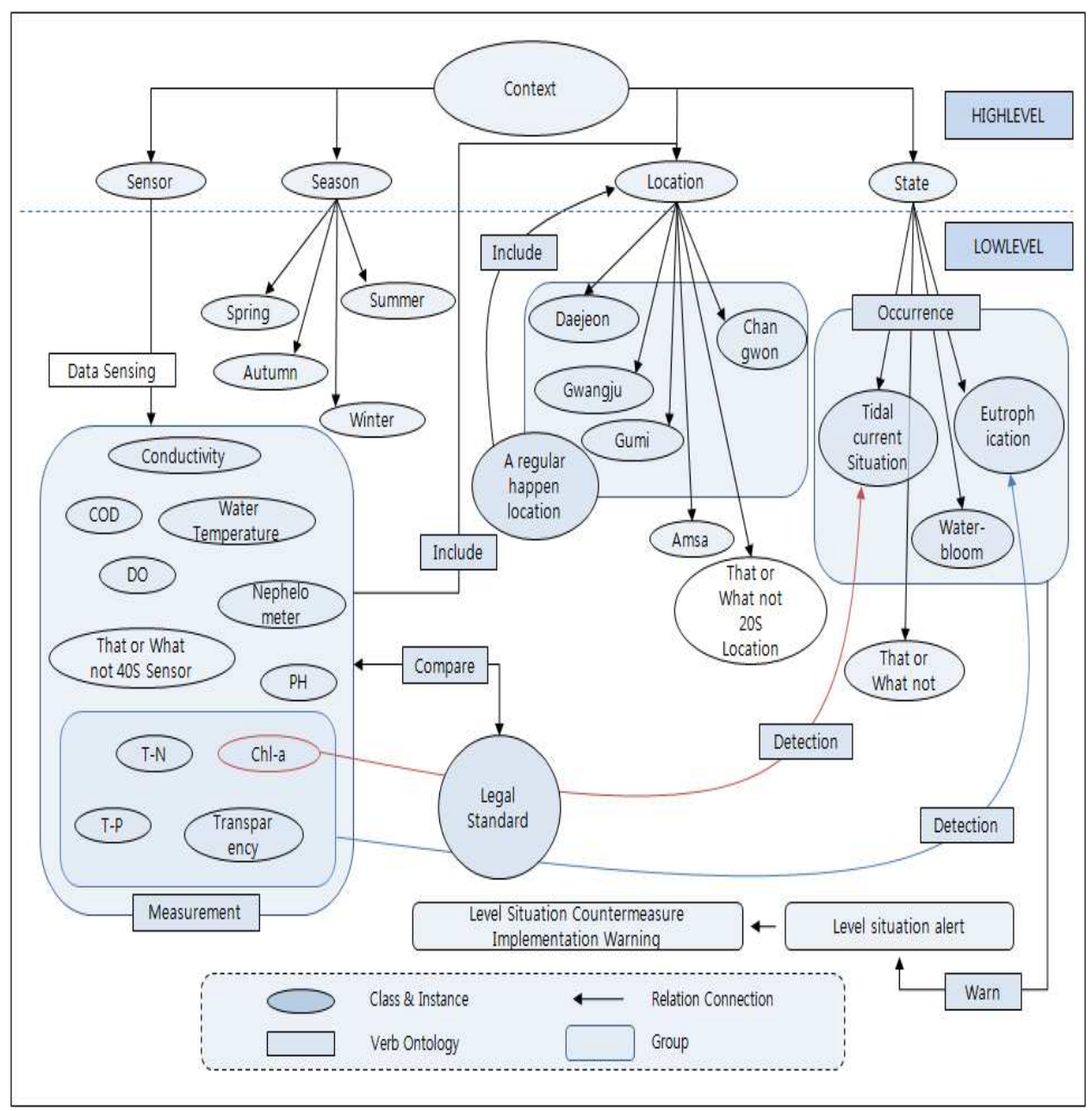

Fig. 4: Context-aware ontology model

In addition, as for chronic occurrent region class which was one of the unmentioned contents, regional location of frequently occurring specific situation at each lake and river is put on the subclass in the location class and represented as "include" relationship to improve the reliability of such researches and to make wetland environment situation awareness more accurate.

Context awareness ontology model application: Various wetland environment items are defined by each word, abnomalties of a specific water quality can be monitored and searched when data exceeds legal standard after reviewing the standard value of relevant items by using the relationship between words that each item has. Table 2 shows the relationship between the data sensed. If eutrophication is usually reached, $\mathrm{PH}$ increases USGS Science for a changing world, Environmental Kit. Eutrophication assessment by four indicators of eutrophication and the variation of numerical value of related items according to the impact of eutrophication can increase the efficiency of environmental management and accuracy of context awareness.

Factual data required for modeling is statistically built in conjunction with distributed data on the web or with data cooperation supported by Water Pure Control Office mentioned above or authoritative agencies. Ontology-based DB table allows you to search information of water pollution possibly to occur by the relationship between terms as quickly as possible.

Table 2: Sensor data correlation

\begin{tabular}{ll}
\hline- & \\
\hline Water temperature increase & DO decrease \\
Water temperature increase & PH decrease \\
COD increase & BOD increase \\
TDS increase & DO decrease \\
\hline
\end{tabular}


Depending on the expansive needs of increasingly automated, intelligent and sensor-driven contextawareness services, awareness of two situations in the management of overall wetland information is enough to prove the effectiveness. In other words, at the time of detection of specific abnormal sensed data, highly associated situations to the item from sensor classes should be recognized, the comparison between season, flow velocity, frequently-occurred region should be made to show the measurement of the possibility, watch and alert level should be provided by recognizing the situation in wetland environments and preventive measures should be taken at the same time of detection of abnormal data and presented to users. In addition, ontology model as above standardizes various representation such as symbols and water terminologies to obtain integrated data, it can be used as indicators for the wetland environment.

The established ontology can rely on other ontologies, thesaurus, dictionaries and be expanded. The utilization of various terms defined in the existing wetland can reduce cost, time and efforts $t$ on the expansion of ontology.

\section{CONCLUSION}

Currently, Water Quality Monitoring Network of Water Pure Control Office only provides monitoring and alarm or generate final elements of water pollution with integrated abnormalties of each item through the opinion of experts.

This study investigates various situations and data related to wetland environment water quality in order to understand user's intention and purpose within the area of wetland management and find the best results. Additionally, this promotes the convenience of the user search by organizing and analyzing a variety of related terms and proposes ontology model in the field of wetland environment, which is the basis of semantic situation detection of the relationship between words.

The proposed domain specific ontology context awareness model is not a deduction of fragmentary values through a simple query but, a provision with lexicons and terms to express and share the situational information as a means of describing concepts and correlations. This model support the share and reuse of situational knowledge in ubiquitous computing environment and creates lower-level of ontology appropriate for specific domain by using upper level ontology as a hierarchical situation ontology model. This provides the basis for the optimization of wetlands which is an important position of man and nature, systematic wetland management of wetland environment related data and data search of dictionary meaning and information.

For the future intelligent data retrieval and detection approach, more diverse subclass and relationship must be defined based on related water quality data collection from the Institute of Public Health and Environment. Further, by applying the proposed ontology model, in addition to information of eutrophication and algae phenomenon and alarm sending, more comprehensive management of wetland environment and continuous research on the methods of how to build and expand applicable to various domain will improve the completeness of system design of wetland environment context awareness.

\section{ACKNOWLEDGMENT}

This research was financially supported by the Ministry of Education, Science Technology (MEST) and National Research Foundation of Korea (NRF) through the Human Resource Training Project for Regional Innovation.

\section{REFERENCES}

Choi, J.H. and Y.T. Park, 2009. A dynamic service supporting model for semantic web-based situation awreness service. KIISE Inform. Sci., 36: 732-748.

Lim, S.Y., M.H. Song and S.J. Lee, 2004. Domainspecific ontology construction by terminology processing. KIISE Korea Inform. Sci., 31: 353-360.

Lim, S.Y., S.O. Koo, M.H. Song and S.J. Lee, 2003. Hub-word based on ontology construction for document retrieval. Proceedings of IC-AI (ICAI' 03), pp: 549-552.

Mahesh, K., 1996. Ontology Development for Machine Translation: Ideology and Methodology. 1st Edn., Computing Research Laboratory, New Mexico State University, Las Cruces, New Mexico, pp: 79.

Park, J. and S. Rarn, 2004. Information systems interoperability: What lies beneath? ACM Trans. Inform. Syst., 22: 595-632. DOI: $10.1145 / 1028099.1028103$

Ranganathan, A. and R.H. Campbell, 2003. A middleware for context-aware agents in ubiquitous computing environments. Proceedings of the ACM/IFIP/USENIX 2003 International Conference on Middleware, Jun. 16-20, ACM, Rio de Janeiro, Brazil, pp: 143-161.

Srinivasan, N., M. Paolucci and K. Sycara, 2006. Semantic web service discovery in the OWL-S IDE. Proceedings of the 39th Annual Hawaii International Conference on System Sciences, Jan. 04-07, IEEE Xplore Press, pp: 109b-109b. DOI: 10.1109/HICSS.2006.431 